\title{
Analyzing Double Image Illusion through Double Indiscernibility and Lattice Theory
}

\author{
Kohei Sonoda ${ }^{1}$, Eugene S. Kitamura ${ }^{1}$, Iori Tani ${ }^{1}$, Tomohiro Shirakawa ${ }^{2}$, \\ \& Yukio-Pegio Gunji ${ }^{1}$
}

${ }^{1}$ koheisonoda@gmail.com, Department of Earth and Planetary Sciences, Graduate School of Science, Kobe University, Nada, Kobe, Japan

${ }^{2}$ Department of Computer Science, School of Electrical and Computer Engineering, National Defense Academy of Japan, Yokosuka, Kanagawa, Japan

\begin{abstract}
The figure-ground division plays a fundamental role in all image perceptions. Although there are a lot of studies about extraction of a figure such as detection of edges or grouping of texture, there are few discussions about a relationship between obtained figure and ground. We focused on double image illusions having two complementary relationships between figure and ground and analyzed them. We divided the double image illusions according to two different interpretations and using these divisions we extracted and analyzed their logical structures by lattices derived from rough sets that we had developed. As a result we discovered unusual logical structures in double image illusions.
\end{abstract}

Keywords: illusion, double images, figure-ground, double indiscernibility, Gestalt theory, rough set theory, lattice theory.

\section{Introduction}

There are various double image illusions, Rubin's vase (Rubin, 1915) being notorious. Double image illusions are said to be possible by the figure-ground perception, established by Gestalt psychology (Koffka, 1935). Figure is the target of attention, and the ground is the background where the figure is contrasted. If the figure or ground status of an object changes, it is called a figureground reversal.

The contrast between figure and ground becomes especially important when interpreting images. Today, interpretation of visual images is becoming more important due to human-computer interface (Huang, 2008) and computer vision (Loss, 2009). Psychological theories are developed for understanding human visual interpretation mechanisms (Huang, 2007).

However, these studies are entirely focused on extraction of the figure such as detection of edges or grouping of texture. The figure-ground relationship obtained in them has almost never been discussed before. We focused on the ambiguity of complementary relationship between figure and ground in double image illusions and analyzed it.

First, we consider the division of a figure image. A two-dimensional figure image is divided into groups by its attributes. In a figure image of a face, attributes are features of the figure image such as eye, mouth, nose, ear, .... When a two-dimensional figure image is divided into several parts, each of them is interpreted as an attribute. We call a mapping from the two dimensional figure to its attribute set as "an interpretation". It is conceivable that two different interpretations are in double image illusions. We define a set of points that are mapped from the figure image to the same attribute as an equivalent class. This equivalent class is obtained by an equivalent relationship derived from each interpretation. So two different sets of equivalent class exist in double image illusions.

To analyze such double-interpretations, we use rough set theory. A rough set is a concept of sets approximated by an equivalent relationship using two kinds of approximation (Pawlak, 1981, 1982). Its definition is the following: Given a universal set $U$ and an equivalent relationship $R \subseteq$ 
$U \times U$, an equivalent class is expressed as $[x]_{R}=\{y \in U \mid x R y\}$ and for $X \subseteq U$ the lower approximation of $X$ is formally defined as $R_{*}(X)=\left\{x \in U \mid[x]_{R} \subseteq X\right\}$ and the upper approximation as $R^{*}(X)=\left\{x \in U \mid[x]_{R} \cap\right.$ $X \neq \phi\}$. We can construct the logical structure (lattice) by collecting fixed points (X such that $\left.R_{*}(X)=X, R^{*}(X)=X\right)$ with these approximations. However, this method can extract only simple logical structures (Boolean lattices) that consist of a combination based on equivalent classes.

We use a lattice driven by pseudo-closure fixed points that we constructed (Gunji \& Haruna, 2010). This is a collection of fixed points derived from a double approximation based on two different equivalent relationships $(S, R)$. The formed lattice will not necessarily be a simple logical structure as mentioned above, since some of the information is lost due to mismatches of the different interpretations. Actually, it is proved that not only Boolean lattices but also arbitrary lattices can be created.

Using this rough set derived lattice we analyzed an ambiguity of the figure-ground relationship in double image illusions.

\section{Methods}

\subsection{Equivalent Relationship and Indiscernibility}

Rough set theory is founded on the idea that the elements in a set are indistinguishable. The indistinguishable elements have an equivalent relationship and belong to the same equivalent class. Let $x, y \in U$ be elements of a universal set $U$ and $f$ be a transformation function. If $f(x)=f(y)$, then $x$ and $y$ have an equivalent relationship. Express the equivalent relationship as $R$. Then equivalent class is expressed as $[x]_{R}=\{y \in U \mid x R y\}$. Thus the lower approximation of $X$ is formally defined as $R_{*}(X)=\{x \in$ $\left.U \mid[x]_{R} \subseteq X\right\}$ and the upper approximation as $R^{*}(X)=\left\{x \in U \mid[x]_{R} \cap X \neq \phi\right\}$.

\subsection{Galois Connection and Lattice}

In a theory of partially ordered set, Galois connection leads to a complete lattice. Given two partially ordered sets $P$ and $Q$, a pair of maps $(F, G)$ with $F: P \rightarrow Q$ and $G: Q \rightarrow P$ is called a Galois connection, if $F(x) \leqq y \Leftrightarrow x \leqq G(y)$ for any $x \in P$ and $y \in Q$. A closure operator $C:=F G: P \rightarrow P$ can be constructed from a Galois connection such that, for any $x, y \in P$, (i) $x \leqq C(x)$; (ii) $x \leqq y \Rightarrow C(x) \leqq C(y)$; (iii) $C C(x)=C(x)$. It means that closure operator is good and natural operator to take a stable structure in a partially ordered set with respect to $F$ and $G$. Overall it results in a (complete) lattice $L_{T}=\{x \in$ $P \mid C(x)=x\}$.

In the context of a rough set, given a universal set $U, R^{*}: P(U) \rightarrow P(U)$ and $R_{*}: P(U) \rightarrow P(U)$ constitute a Galois connection. Actually, for any $X, Y \subseteq U, R^{*}(X) \leqq Y \Leftrightarrow X \leqq R^{*}(Y)$. Thus $C=R^{*} R^{*}$ is defined as a closure operator and $L_{C}=\{X \in U \mid C(X)=X\}$ is a complete lattice. This lattice, however is too trivial to observe the structure, since it is destined to be a set lattice, thus for any $A \in L_{C}$, complement of $A$ is defined as $A^{C}=U-A$.

If two kinds of binary relationships $R$ and $S$ are on a universal set $U$, and two kinds of operations $S_{*}$ and $R^{*}$ (or $S^{*}$ and $R_{*}$ ), a pair of operations do not constitute a Galois connection. Indeed, if an operator $T=R_{*} S^{*}$ is introduced, $T$ is not a closure operator since it satisfies only (ii) $X \subseteq Y \Rightarrow T(X) \subseteq$ $T(Y)$ and (iii) $T T(X)=T(X)$ for $X, Y \subseteq U$. We call this operator pseudo-closure. If fixed points with respect to pseudo-closure are collected by $L_{T}=\{X \in U \mid T(X)=X\}, L_{T}$ is a lattice but not a set lattice. Inversely, it can be verified that any lattice is expressed in the form of $L_{T}$ by determining adequate equivalent relationships $S$ and $R$. It gives suitable results, reflecting the two different interpretations of illusionary images. 


\subsection{Constructing a Lattice}

Lattice is an algebraic structure where any two elements of a partially ordered set have a unique least upper bound (join) and a greatest lower bound (meet) (Birkhoff, 1967; Davey \& Priestley, 2002). As mentioned before, given two kinds of equivalent relationship $R$ and $S$ on a universal set $U$, we can construct a lattice by $\left\langle L_{T} ; \subseteq>\right.$ with $L_{T}=\{X \subseteq U \mid T(X)=X\}, T=R_{*} S^{*}$. Actually, an element of $L_{T}$ is a subset of the universal set, and order is defined by inclusion $\subseteq$. If all subsets of $U$ are collected, $L_{T}$ is a power set, and is a set lattice in which join and meet are defined by union $U$ and intersection $\cap$, respectively. In general, $L_{T} \neq \mathscr{A} U$ ), thus join and meet are defined by the following: for any $X, Y \in L_{T}, X \wedge Y=T(X \cap Y), X \vee Y=T(X \cup Y)$. It can be verified that $L_{T}$, is closed with respect to $\vee$ and $\wedge$, and that $L_{T}$ is a lattice. We call $L_{T}=\{X \in U \mid T(X)=X\}$, with $T=R_{*} S^{*}$.

When we construct a lattice of fixed points from one equivalent relationship, $R_{*}\left(R^{*}(X)\right)=X$, we only get a set lattice. A set lattice has two important properties in lattice theory such as distributivity, $A \wedge(B \vee C)=(A \wedge B) \vee(A \wedge C)$ for $A, B, C \subseteq U$, and complementarity of which for any $X \subseteq U$, there exists $Y \subseteq U$ such that $X \vee Y=U, X \wedge Y=\phi$. Note that $U$ and $\phi$ are the greatest and least element in the lattice $L_{T}$. A distributive complemented lattice is called a Boolean lattice. However, when we construct a lattice of fixed points from two equivalent relationships, $R_{*}\left(S^{*}(X)\right)=X$, the resulting lattice can be either a Boolean lattice or a non-Boolean lattice. This is a result of two equivalent classes fully or partially overlapping each other. The differences between Boolean and non-Boolean lattices are mentioned in the next section. Difference between a lattice of $L_{C}=\{X \subseteq U \mid C(X)=X\}$ and $L_{T}=\{X \subseteq$ $U \mid T(X)=X\}$ with $C=R_{*} R^{*}, T=R * S^{*}$ is illustrated in Fig. 1. In the case of $L_{C}$, when we denote $\left.W=U / R=\left\{[a]_{R},[c]_{R},[d]_{R}\right\}=\{\{a, b\},\{c\},\{d, e\}\}, L_{C}=\not A W\right)$, where all possible combinations of equivalent class of $R$ are obtained (Fig. 1 (a)). By contrast, in $L_{T}$, although elements of $L_{T}$ are possible unions of equivalent class $R$, some elements are missing (Fig. 1 (b)). Actually, $R_{*} S^{*}(\{a, b, c\})=R_{*}\left([a]_{S} \cup\right.$ $\left.[b]_{S}\right)=R_{*}(\{a, b, c, d\})=[a]_{R} \cup[c]_{R}=\{a, b, c\}$. Thus $\{a, b, c\}$ is an element of $L_{T}$, on one hand. On the other hand, since $R_{*} S^{*}(\{a, b, d, e\})=R_{*}\left([a]_{S} \cup[b]_{S} \cup[e]_{S}\right)=R_{*}(U)=U \neq\{a, b, d$, e\}, hence $\{a, b, d, e\}$ is missing in $L_{T}$. Due to the loss of information, an obtained lattice can be constructed as a nonBoolean lattice. 


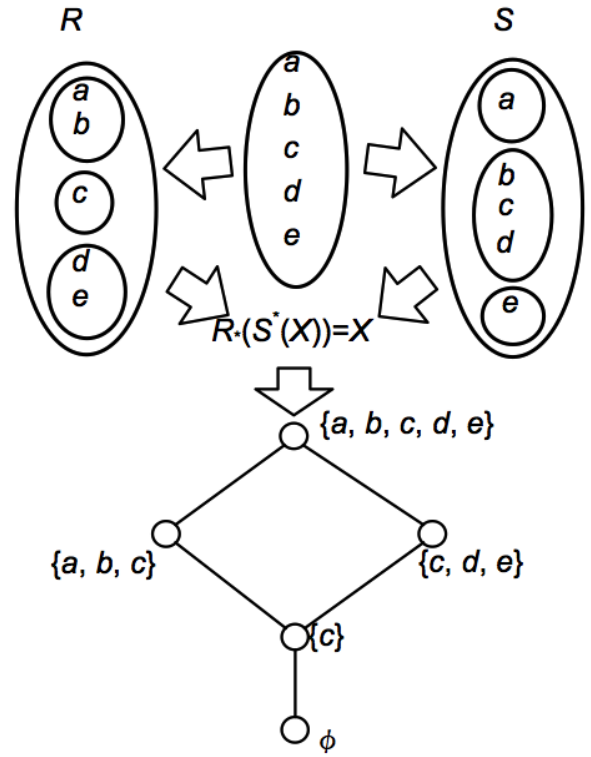

(a)
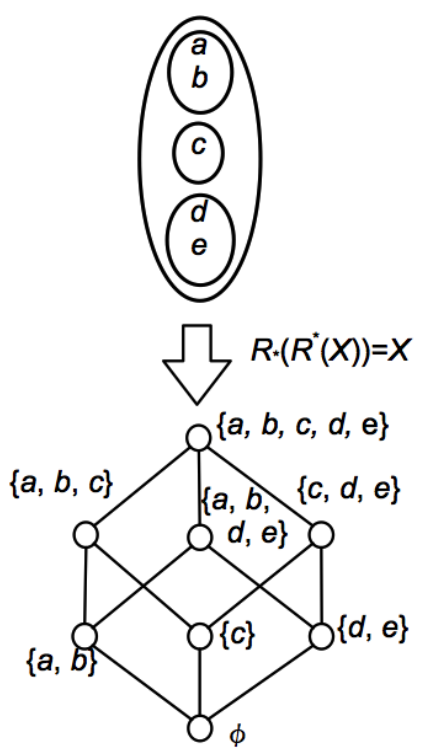

Figure 1: (a) Only Boolean lattices result when using fixed points from a single equivalent relationship. (b) Boolean as well as non-Boolean lattices result when using fixed points from double equivalent relationship. The lattice shown here is a non-Boolean lattice. Elements $\{a, b\},\{d, e\}$, and $\{a, b$, $d, e\}$ from (a) are missing in (b).

To construct a rough set derived lattice from two equivalent relationships, we need two interpretations, $R$ and $S$. Each fixed point $X$ considered is an equivalent class, for example $\{a, b\}$ or $\{c\}$ or $\{d$, e\} in Fig. 1 (b) for the interpretation $R$. Treating each equivalent class as a unit, we consider its power set: $\phi,\{a, b\},\{c\},\{d, e\},\{a, b, c\},\{c, d, e\},\{a, b, d, e\}$, and $U=\{a, b, c, d, e\}$. Each equivalent class and its power set composition are used as an $X$, and operators $S^{*}$ and $R^{*}$ are applied in this order. When applying $S^{*}$ to the $X$ of the interpretation group $R$, one must take the upper approximation of $X$ in terms of interpretation $S$ since we are applying $S^{*}$. For example, take $\{c\}$ of interpretation $R$ and apply $S^{*}$. We get $S^{*}(X)=\{b, c, d\}$. Next, apply $R_{*}$ to $S^{*}(X) . R_{*}(\{b, c, d\})$ is $\{c\}$ in interpretation $R$, since only the equivalent class $\{c\}$ is included completely within the elements $\{b, c, d\}$. We started with $X=\{c\}$ and we get $R_{*} S^{*}(X)=\{c\}$. Therefore, the equivalent class $\{c\}$ is considered a fixed point. If we take $\{a, b\}$ in terms of interpretation $R$ and apply $S^{*}$ and follow the same procedure, we get $R * S^{*}(X)=\{a, b, c\}$. Therefore, the equivalent class $\{a, b\}$ is not a fixed point. Repeat this process for all element sets in the power set, from $\phi$ to $U$. The empty set $\phi$ and the universal set $U$ will always be a fixed point. Then collect the fixed points and use them as elements to build a lattice based on its inclusion relationships, with the universal set at the top and the empty set $\phi$ at the bottom.

\subsection{Relation Table}

The relationship between the two equivalent relationships $R$ and $S$ is expressed by using a relation table shown in Table 1. We use such tables to organize the information and to find fixed points to construct rough set derived lattices as explained in the previous procedure section (2.4). The example we show here uses the two equivalent relationships $R$ and $S$ in Fig. 1 (b). Table 1 shows the relationship between the elements of the equivalent relationships $R$ and $S$. 1's denote the presence 
of a relationship and 0's denote the lack of a relationship. In subsequent sections, we will refer to such relation tables.

\begin{tabular}{|c|c|c|c|c|c|c|}
\hline \multicolumn{2}{|c|}{} & \multicolumn{6}{|c|}{$S$} \\
\cline { 2 - 7 } & $a$ & $b$ & $c$ & $d$ & $e$ \\
\hline \multirow{4}{*}{$R$} & $a$ & 1 & 1 & 1 & 1 & 0 \\
\cline { 2 - 7 } & $b$ & 0 & 0 & 0 & 0 & 0 \\
\cline { 2 - 7 } & $c$ & 0 & 0 & 1 & 1 & 0 \\
\cline { 2 - 7 } & $d$ & 0 & 0 & 0 & 1 & 1 \\
\hline & $e$ & 0 & 1 & 1 & 1 & 1 \\
\hline
\end{tabular}

Table 1. A relation table showing the relationships between $R$ and $S$ according to the elements

\section{Procedures}

\subsection{Experiment}

Six different double image illusions were used as samples (Fig.2). Double image illusions were printed on A4 papers in monochrome. The randomly ordered samples were provided to the subjects for the task. Thirty-one individuals participated in the experiment. The subjects were healthy individuals in their 20 s and 30s. The experiment room was approximately 20 degree $C$ and other conditions such as lighting were kept constant. When marking the sheet, the subject was allowed to turn it or move it freely as long as it stayed flush to the plane of the table. The color of the ink was pink and the line diameter was $5 \mathrm{~mm}$.

The task of the experiment was to mark six attributes of a double image illusion. We instructed a subject to view the image as one of the two interpretations of the double image illusion. Before starting with the task, the subjects were checked whether or not they recognized the instructed interpretation. If they did not recognize it, we explained it without pointing to any parts or attributes. In a case where the subject recognized the image, the task was started. If this was not the case, the task for this image and a task according to another interpretation of the double image illusion were skipped. An attribute of an image may be an eye, nose, ear, neck, shoulder, or any aspect of an image that the subject especially noticed. These attributes were enclosed with a marker. The shape and size of the enclosure were arbitrary. However, lines of two different enclosures could not touch. The order of the six images and either of the two interpretations were randomly determined. The two interpretations were marked consecutively on different sheets of paper.

After the experiment, the two sheets from the two interpretations were compared for overlap in the attribute enclosures (Fig.3). When they overlapped, they were considered to have a relationship. An intersection of lines is not counted as an overlap and we considered only an intersection of inner portion of the enclosures. An enclosure on one interpretation may have more than one attribute overlap with the other interpretation. With these relationships, a relation table was made as in Table 1 and 2. The procedure (section 2.4) was followed to construct a lattice. 


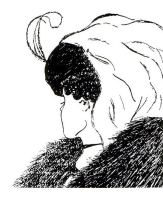

(a)

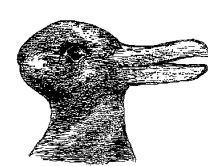

(b)

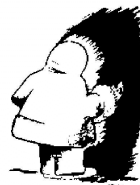

(c)

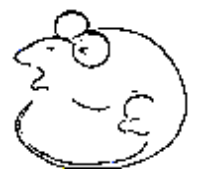

(d)

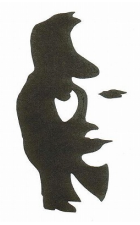

(e)

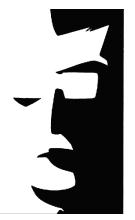

(f)

Figure. 2: Images used for the experiment: (a) young lady vs. old lady, (b) duck vs. rabbit, (c) indigenous tribe member vs. Inuit, (d) bald man vs. mouse, (e) lady vs. saxophone player, (f) front view of a man's face vs. side view of a witch's face (by Yuya Maekawa 2008).

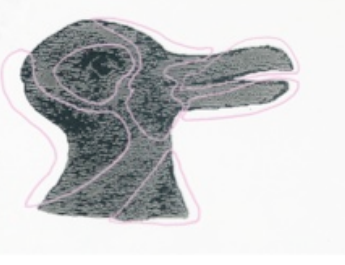

(a)

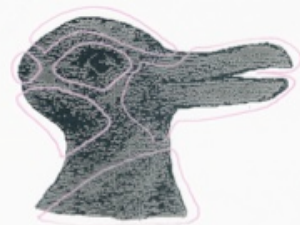

(b)

Figure 3: divisions of duck (a) and of rabbit (b).

\begin{tabular}{|c|c|c|c|c|c|c|c|}
\hline \multicolumn{2}{|c|}{} & \multicolumn{7}{|c|}{$S$} \\
\cline { 2 - 8 } & $a$ & $b$ & $c$ & $d$ & $e$ & $f$ \\
\hline \multirow{4}{*}{$R$} & $a$ & 1 & 0 & 0 & 0 & 0 & 0 \\
\cline { 2 - 8 } & $b$ & 1 & 0 & 0 & 0 & 0 & 0 \\
\cline { 2 - 8 } & $c$ & 1 & 1 & 0 & 0 & 0 & 0 \\
\cline { 2 - 8 } & $d$ & 0 & 0 & 1 & 0 & 0 & 0 \\
\cline { 2 - 8 } & $e$ & 0 & 0 & 0 & 1 & 1 & 0 \\
\hline & $f$ & 0 & 0 & 0 & 0 & 0 & 1 \\
\hline
\end{tabular}

Table 2: The relation table of duck vs. rabbit of Fig.3.

\subsection{Control}

As a control for the experiment, we created two sets of equivalent classes by Monte Carlo method and a rough set derived lattice by overlapping them. The number of trials was 100,000 and that of equivalent classes was six. We used a grid of $100 \times 100$ and followed the algorithm below.

- Step 1 Assign 0 to all cells in the grid (initialization).

- Step 2 Assign values from 1 to 6 to randomly chosen cells. These are starting points of the equivalent classes. 
- Step 3 Choose the number of maximum iteration (maximum growth) randomly between 1 to 100 and repeat the following step as many times as the maximum iteration.

- Step 4 Cells are processed from $(0,0)$ to $(99,99)$ in the $x$-direction first. If the cell $(x, y)$ has a value other than 0 , choose a cell randomly from $(x+1, y),(x-1, y),(x, y+1),(x, y-1)$. If the randomly chosen new cell has a value 0 , assign the same number as $(x, y)$.

\subsection{Analysis}

For analyzing the derived lattices, we defined a figure and a ground by a complementary relationship. In other words an equivalent class a has the figure-ground relationship with $b$ when they satisfy $a \vee b=1$ and $a \wedge b=0$. As a simple example, we consider Rubin's vase. When $a=$ "vase" is the figure and $b=$ " two faces" is the ground, it can be said that $a$ and $b$ together express the whole image (Rubin's vase). Therefore, by investigating complementary relationships, we can analyze the logical structures (the derived lattices) in terms of the figure-ground relationship.

Furthermore we introduce non-distributivity (ND) and complementarity (C) as barometers for the analysis. This is because an equivalent class may have more than one complement. We define ND by complement possession rate and $C$ by complement existence rate. ND does not count an element that has no complements. So we get $N D>=1.0$. From this definition, if the derived lattice is a distributive one, then we get $N D=1.0$ (one-to-one complementary relationship). If it is a complementary one, then we get $\mathrm{C}=1.0$ (all elements have some complements). Therefore this definition is consistent with lattice theory. However, a distributive lattice doesn't necessarily imply ND=1.0. Yet less than one percent of all lattices satisfied $N D=1.0$ and were distributive in the experiment. On the other hand, a complementary lattice and $\mathrm{C}=1.0$ are equivalent. Thus, we can say that this definition is effective.

For example, we consider a lattice $\{\phi, a, b, a b\}$. Complementary pairs are $\phi-a b$ and a - b. So we get $\mathrm{ND}=1.0$ since each element has only one complement and $C=1.0$ since each element has a complement. In fact, this is a Boolean lattice since $C=1.0$ and $N D=1.0$. For the next example, we consider a lattice $\{\phi, a, b, c, a b c\}$. Complementary pairs are $\phi-a b c, a-b, b-c$, and c - a. Obviously $C=1.0$. But this differs from the previous example in that $N D>1.0$ since some elements have more than one complement. In other words, $N D=8 / 5=1.6$ since complementary pairs are $8(\phi-$ abc, a - b, a - c, b- a, b - c, c - a, c - b, and abc- $\phi$ ) and the number of elements with complements are $5(\phi, a, b, c$, and abc). So, this is not a distributive lattice but a complementary one since $\mathrm{C}=1.0$.

\section{Results}

We must be careful about the size of the obtained lattices when we analyze their non-distributivity and complementarity. The number of elements determines the size of a lattice. If it is small, the possibility of an element's being a complement or complementarity will drop to a lower value and complementary relationships will be one-to-one. In other words, the smaller the size of lattices, the greater the chance of increasing its non-distributivity. So we must restrict the size of the lattices.

For simplicity, we define the size of the lattice by "relation size" (i.e., the number of rows with values other than 0 times the number of columns with values other than 0 ). For example, the relation size of unit matrix with 6 degrees is $36(=6 \times 6)$. In the experiment and the control, the value of the relation size will be between 0 and 36 since the number of equivalent classes is 6 . In this paper we limited the relation size to more than 30 . In other words, the relation size will be 30 or 36 and relation table composition will be $5 \times 6,6 \times 5$, or $6 \times 6$.

The number of samples that satisfy the condition of the relation size was 119 in the experiment and 92,697 in the control. We calculated C and ND of these samples and made a comparison between the experiment and the control by using the relative frequency distribution of these values (Fig3). 
In the control, both $\mathrm{C}$ and ND were distributed widely (Fig3.a). In the experiment, $\mathrm{C}$ was distributed widely and ND was distributed near 1.0 (Fig3.b). Concerning C, the average was 0.6578 and the variance was 0.06978 in the control, and the average was 0.7757 and the variance was 0.05367 in the experiment. Concerning ND, the average was 1.331 and the variance was 0.1391 in the control, and the average was 1.055 and the variance was 0.03063 in the experiment. In Student's ttest, the comparison showed significant differences in both $\mathrm{C}$ and ND at 1 percent (t-value was 4.830 at $C$ and 8.074 at ND).

Non-distributivity of the experiment was remarkable. The average was 1.055 and 80.67 percent for all those that satisfied ND=1.0 (79.83 percent were distributive lattices). In the control, the average was 1.331 and 29.94 percent of all satisfied $N D=1.0$. Results showed that logical structures of double image illusions were more distributive than that of the control.

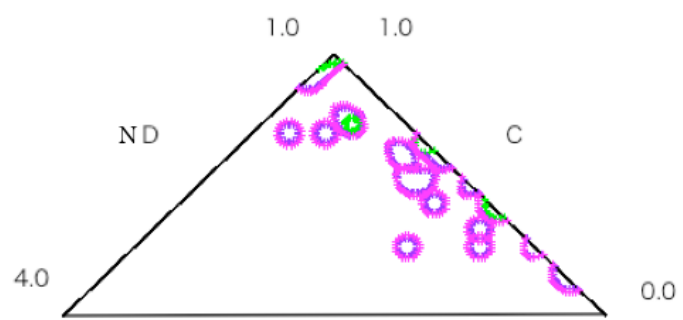

(a)

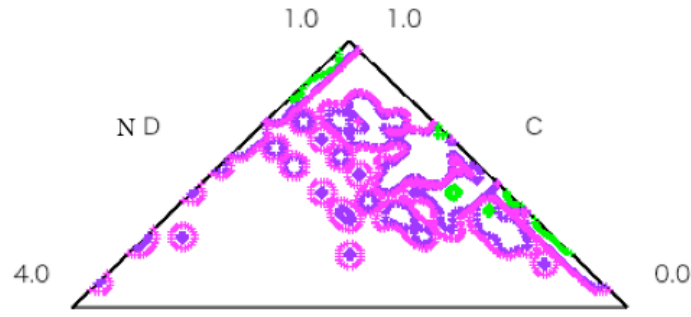

Figure 4: (a) Control (b) Experiment. The heights of the relative frequency distribution contour lines are 0.0002 (purple), 0.002 (blue), 0.02 (green).

\section{Discussion}

The figure and the ground are respectively the region emerging as a shape and the region perceived as its background. It is said that a figure image is divided into the figure and the ground when the figure recognition is realized. For example, we consider Rubin's vase. It can be said that the figure-ground division corresponds to one type of recognition though the figure-ground reversal may emerge. "Vase" becomes the figure and the other parts ("two faces") become the ground when it is perceived and vice versa.

It is true about not only the shape of the figure images but also each division of them. For example, we consider the divisions of a face figure. Let the divisions be \{eyes, nose, mouth, ear, chin, hair\}. When \{eyes\} is attended, it becomes the figure and \{nose, mouth, ear, chin, hair\} becomes the ground. Or, when \{nose, mouth\} is the figure, \{eye, ear, chin, hair\} becomes the ground. In other words, when the shapes or the divisions of the figure are recognized, the figure and the ground are completely divided. At this time, the figure corresponding to the recognition decides the ground. So, when the recognition, namely, the figure-ground division is realized, the figure-ground relationship is one-to-one.

In this paper, the figure-ground relationship is defined by the complementary one. However, the complementary relationship is not always one-to-one. Especially, it becomes one-to-many when its logical structure is not a distributive lattice. This means that the figure-ground relationship is not one-to-one, namely, the figure-ground division is not realized. Otherwise, when the logical structure is a distributive lattice, the complementary relationship, namely, the figure-ground relationship is one-to-one. At this time, the figure-ground division is realized.

From the experimental result, the possibility of obtaining a distributive lattice for the logical structures of double image illusions is very high. But the possibility of a distributive lattice is low for the control. Thus, it can be thought that the logical structures of double image illusions realize the fig- 
ure-ground division and this is not seen in the control. Though both have two different interpretations, it can be thought that only double image illusions have special logical structures realizing the figure-ground division, namely, distributive lattices.

Then, what is the origin of distributive lattices? It comes from ambiguity itself. Ambiguity has meaning in both two different interpretations and cannot be ignored. If you cover ambiguity points by equivalent classes derived from both interpretations, you cannot avoid including the relationships among equivalent classes. For example, let us see "young lady vs. old lady" as the double image illusion. Let there be two equivalent classes such as $\{\{$ eyes $\}$, \{nose $\}\}$ in "young lady" interpretation. Then consider how to cover these two ambiguous points in "old lady". We may cover them by one equivalent class such as \{right eye\}. Or, we can cover them by a bigger equivalent class such as \{both eyes\}. In either case, through these ambiguous points, the including relationships such as $\{$ eye $\},\{$ nose $\} \subseteq$ right eye $\}$ or $\{$ eye $\},\{$ nose $\} \subseteq\{$ both eyes $\}$ are formed between equivalent classes derived from two interpretations. Of course, it can be possible that equivalent classes from one interpretation include that from another and vice versa. When all equivalent classes in both interpretations are in some including relationships, their logical structure becomes a Boolean lattice. If some parts deviate from that case, divisions of double image illusions will be separated into regions with including relationships and regions with entanglements. Nondistributivity comes from these entangled regions that form non-distributive lattices $\left(N_{5}\right.$ or $\left.M_{3}\right)$ as sub-lattices. But, the possibility to generate non-distributive lattices is generally small since the sublattices have small logical structures (relation size).

Thus, if there are some regions with partial including relationships in double image illusions, the possibility for their logical structures to become distributive lattices is very high. It can be said that ambiguity generates distributive lattices as a logical structure. Of course, this cannot be seen in the control since it has randomness and doesn't have such ambiguity.

\section{References}

Birkhoff, G. (1967). Lattice Theory. Coll. Publ., XXV, American Mathematical Society, Providence.

Davey, B.A. \& Priestley, H.A. (2002). Introduction to Lattice and Order, 2nd ed., Cambridge University Press, Cambridge.

Fig. 2 (a) Lucks Inc. [a blog, in Japanese], http://www.lucks.tv/blog/455.html

Fig. 2 (b) Juggler News [a blog, in Japanese], http://montafresh.exblog.jp/9708641/

Fig. 2 (c) Lalala Meditation [a blog, in Japanese], http://plaza.rakuten.co.jp/vijay/diary/200507060001/

Fig. 2 (d) Uguisu No Kimyouna Seikatsu [a blog, in Japanese], http://ugisu.blog71.fc2.com/blog-category-9.html

Fig. 2 (e) Kokoro To Karada No lyashi No Message (A Healing Message for the Heart and the Body) [a blog, in Japanese], http://kkmessage.exblog.jp/9029398/

Fig. 2 (f) Tayama Lab., Department of Psychology, Hokkaido University, http://www3.psych.let.hokudai.ac.jp/tool/maekawa/ojisamahamajo.gif

Gunji, Y.-P. \& Haruna, T. (2010). A Non-Boolean Lattice Derived by Double Indiscernibility. Transactions on Rough Sets XII, LNCS, 6190, 211-225.

Huang, K.-C. (2008). Effects of computer icons and figure/background area ratios and color combinations on visual search performance on an LCD monitor. Displays, 29(3), 237-242.

Huang, L. \& Pashler, H. (2007). A Boolean Map Theory of Visual Attention. Psychological Review, 114(3), 599-631.

Koffka, K. (1935). Principles of Gestalt Psychology. London: Routledge \& Kegan Paul.

Loss, L., Bebis, G., Nicolescu, M. \& Skurikhin, A. (2009). An iterative multi-scale tensor voting scheme for perceptual grouping of natural shapes in cluttered backgrounds. Computer Vision and Image Understanding, 113(1), 126-149.

Pawlak, Z. (1981). Information systems-theoretical foundations. Information Systems, 6, 205-218.

Pawlak, Z. (1982). Rough Sets. International Journal of Computer and Information Science, 11, 341-356.

Rubin, E. (1915). Synsoplevede Figurer. Gyldendal, Koebenhavn.

\section{About the Authors}

Kohei Sonoda

is a postdoctoral fellow at the University of Kobe, Japan. He earned a Ph.D. degree in Science from the University of Kobe. $\mathrm{He}$ is the author of other articles and special research reports on computer science and animal behavior.

Eugene S. Kitamura

is a postdoctoral fellow at the University of Kobe. He earned a Ph.D. degree in Science from the University of Kobe. He is the author of other articles and special research reports on literary text analysis. 
Iori Tani

is currently working towards a Ph.D. degree in Science at the University of Kobe. He earned an M.A. degree in Science from the University of Kobe.

\section{Tomohiro Shirakawa}

is a research associate at National Defense Academy of Japan. He earned a Ph.D. degree in Science from the University of Kobe. He is the author of many articles and special research reports on cell biology (of the plasmodium of Physarum polycephalum), emergent phenomena in biological systems and nature-inspired bio computing.

\section{Yukio-Pegio Gunji}

is a professor of theoretical bioscience at the University of Kobe. He is the author of many articles and special research reports on philosophy, mathematics, computer science, chemical biology, cognitive science, animal behavior and economics. 